$\$=$

\title{
Risk factors and management of breast cancer-related lymphedema
}

\author{
Ayat Omer Ibrahim ${ }^{1}$, Awad Ali M. Alawad ${ }^{2 *}$ \\ ${ }^{1}$ Specialist general surgeon, Khartoum, Sudan \\ ${ }^{2}$ Assistant Professor of Surgery, Faculty of Medicine, University of Medical Sciences Technology, Khartoum, Sudan \\ *Corresponding author E-mail:awadali82@hotmail.com
}

\begin{abstract}
Background: Lymphedema is a highly prevalent condition in women who have undergone treatment for breast cancer. Lymphedema negatively affects the quality of life.

Objective: The aim of this study was to identify the clinical presentation of lymphedema associated factors in women treated for breast cancer.

Methods:The study is an interventional prospective study. It was accomplished in the period from May 2013 to April 2014. Patients having breast cancer ipsilateral arm lymphedema were selected for the study.

Results: A total of 34 patients were included in the study.73.5\% of patients have a body mass index (BMI) of 25 or more at diagnosis. $70.6 \%$ of the patients underwent axillary clearance. All the patients did not undergo breast reconstruction. $52.4 \%$ had $<10$ lymph nodes removed from their axillae. $47 \%$ received radiotherapy. All patients presented with arm swelling, additionally, $70.6 \%$ presented with arm heaviness $26.5 \%$ presented with arm pain. Elevation was effective in $90.5 \%$ of the compliant patients, while exercise was effective in $84.2 \%$ of the compliant patients.

Conclusion: Breast cancer- related lymphedema continues to be a significant problem following breast cancer therapy. Presence of comorbid conditions axillary radiation significantly increases the risk of lymphedema. A combination of axillary dissection axillary radiation should be avoided whenever feasible to avoid lymphedema.
\end{abstract}

Keywords:Lymphedema, Risk Factors, Breast Cancer.

\section{Introduction}

Breast cancer continues to be the most frequently occurring cancer in women (Ahmed, 2014). With the advent of multimodality treatment early detection methods, there is an overall improvement in survival. With this transformation of the disease into a chronic condition the focus of attention is recently being directed towards late post treatment sequelae like lymphedema.

Lymphedema in patients with breast cancer is caused by interruption of the axillary lymphatic channels by surgery /or radiation therapy (Monleon et al., 2014). Axillary dissection radiation therapy has a synergistic effect on lymphedema risk which results in the accumulation of fluid in subcutaneous tissue in the arm, with decreased distensibility of tissue around the joints increased weight of the extremity. Lymphedema may present immediately or years after axillary dissection (Marcos et al., 2012). It has been reported as late as 30 years after treatment. Although the incidence is generally accepted at approximately $20 \%$, reported rates vary greatly, ranging between 6 - 30\% (van der Veen et al., 2004).

Lymphedema is regarded as a progressive, disfiguring, disabling disorder that is difficult to treat; therefore it is essential to prevent or minimize this condition. The aim of this study is to determine patient- treatment-related risk factors of lymphedema in breast cancer patients.

\section{Patients and methods}

The study is an interventional prospective study. It was accomplished in the period from May 2013 to April 2014 at Khartoum oncology centre. Patients with breast cancer ipsilateral arm lymphedema were selected for the study. Arm circumference was measured in two points; one at the wrist the other at the elbow joints. Measurement was done using tape was done by the researcher herself. Lymphedema was diagnosed if there was increase by $2 \mathrm{~cm}$ or more at one point at least in excess of the affected arm. If it was less than $2 \mathrm{~cm}$, patient was excluded from the study.

Patients were inquired about the treatment modalities received before, if any, whether they were effective. Arm elevation, exercise, skin care instructions were discussed with the patient in details. Patients were advised regarding modulation of home activities tight finger rings bracelets avoidance. Arm elevation was achieved during sleep by putting the arm over a pillow during the day time by arm sling application. Application effects of crepe bandaging were discussed with all patients.

Patients who were compliant to crepe bandage application were taught about it the first application was done by the researcher. The crepe bandage was used in addition to the exercise elevation. There were no restrictions regarding timing of application.

After a period ranging from four to forty three days, the majority of patients were followed up regarding the subjective response. For patients who came for the follow up, new arm circumference 
measurement were taken compared with the previous readings. If patient died or didn't come back this was recorded.

Data were entered into a Microsoft Excel spreadsheet the statistical analysis was conducted using SPSS (Version 17). Statistical analysis was carried out using descriptive analytical statistics. Simple frequencies cross tabulation were done. Chi square test was used for proportions. Stratification for the patient's sex educational level was done when relevant. $\mathrm{P}$ value of less than 0.05 was considered statistically significant.

The consent of the patients was obtained. A full explanation of the purposes nature of the study was conveyed to them. The potential participants were clearly assured that their participation in this study is voluntary any data obtained would be treated confidentially for the purpose of the research only.Results

A total of 34 patients were seen during the period May 2013 April 2014. It was found that $38.2 \%$ of patients are $40-49$ years old that only $14.7 \%$ of them are 60 years or above. $38.2 \%$ of patients $(n=13)$ are living in rural areas while $61.8 \%$ of them $(n=21)$ are living in urban areas. In our study $73.5 \%$ of patients $(n=25)$ have body mass index 25 or more $26.5 \%$ of them $(n=9)$ have BMI $<25$ (Tab. 1).

It was found that $44.1 \%$ of patients $(n=15)$ have no report regarding their nodal status at diagnosis; $32.4 \%$ of patients $(n=11)$ had $\mathrm{N} 1$ status at diagnosis $8.8 \%$ had $\mathrm{N} 2$ or $\mathrm{N} 3$ at diagnosis. We found that $73.5 \%(\mathrm{n}=25)$ underwent axillary clearance; the remaining either did not undergo axillary operation or the operation details were not reported. $11.8 \%$ of patients $(n o=4)$ did not undergo breast operation. $588 \%$ of patients $($ no $=20)$ underwent modified radical mastectomy. $52.4 \%$ of our patients $(n=11)$ had $<10$ lymph nodes removed from their axillae. $76.2 \%$ of patients $(n=16)$ had $<10$ affected lymph nodes after the axillary operation (Tab. 2 and 3). $44.1 \%$ of patients $(n=15)$ did not receive radiotherapy (RT) at all.

All thirty four patients presented with limb swelling, in addition many patients presented with heaviness /or pain. Duration of symptoms is ranging from 0.8 to 5.0 years with a mean of 1.24 years. Examination of patients revealed that 33 of them have stage II only one has stage III lymphedema. Wrist circumference elbow circumference was measured bilaterally for all patients. The increase in the circumference of the affected limb was calculated in percentage; for the wrist the increase was ranging from $0 \%-70 \%$ the mean was 12.03 . For the elbow the increase was $2-78 \%$ the mean was $15.39 .85 .3 \%$ of patients $(n=29)$ were taught about elevation before $79.4 \%$ of patients $(n=27)$ were taught about exercise before. 21 out of the 29 patients were compliant to elevation.19 patients out of the $21(90.4 \%)$ said that it is effective two said that it is not effective. 19 out of the 27 patients were compliant to exercise. 16 patients out of the $19(84.2 \%)$ said it is effective 3 said it is not effective. Only four out of the thirty four patients were told about bandaging before, all of them used it and all of them claimed that it is effective. Elevation was effective in $90.5 \%$ of the compliant patients, while exercise was effective in $84.2 \%$ of the compliant patients. It was found that $100 \%$ of the compliant patients for bandaging said that it is effective, whether they were instructed by the researcher or somebody else. All patients who responded to bandaging were followed up verbally regarding its subjective effect; they all claimed that it is effective in swelling reduction.

Only four of the patients for whom crepe bandaging was applied were followed up objectively, because some patients died and some refused to come because they are living far away or because they feel well and no need to come back. The four patients were followed up within a period ranging from four to eighty nine days. All of them showed reduction in the circumference for both the elbow and the wrist. For the wrist the reduction percentage range was $1.8-31 \%$ and the mean was13.9. For the elbow the reduction percentage range was $2.6-42.9 \%$ and the mean was 17 .
Table 1: Showing the Body Mass Index of the Patients $(\mathrm{N}=34)$

\begin{tabular}{lll}
\hline & Frequency & Percent \\
\hline$<20$ & 2 & 5.9 \\
$20-24$ & 7 & 20.6 \\
$25-29$ & 13 & 38.2 \\
$>30$ & 12 & 35.3 \\
Total & 34 & 100.0 \\
\hline
\end{tabular}

Table 2: Showing Number of Removed Lymph Nodes $(\mathrm{N}=34)$

\begin{tabular}{lll}
\hline & Frequency & Percent \\
\hline$<10$ & 11 & 52.4 \\
$10-19$ & 6 & 28.6 \\
$20-29$ & 3 & 14.3 \\
$>30$ & 1 & 4.8 \\
Total & 21 & 100.0 \\
\hline
\end{tabular}

Table 3: Showing Number of Affected Lymph Nodes (N=34)

\begin{tabular}{lll}
\hline & Frequency & Percent \\
\hline 0 & 8 & 38.1 \\
$1-9$ & 8 & 38.1 \\
$10-19$ & 2 & 9.5 \\
20 or more & 3 & 14.3 \\
Total & 21 & 100.0 \\
\hline
\end{tabular}

\section{Discussion}

We found that $38.2 \%$ of patients are $40-49$ years old that only $14.7 \%$ of them are 60 years or above. This does not seem to be in accordance with the literature because it was found that lymphedema (LE) incidence increases with age. However, this might be affected by the age of patients with breast cancer in $\mathrm{Su}$ dan (A. A. M. Alawad, 2014). Sudanese patients with breast cancer are younger than other country's' patients (A. Alawad, Alshiekh, \& Alhaj, 2014). In our study $73.5 \%$ of patients (no=25) have body mass index of 25 or more. This result is in accordance with the reported literature; it was found that there is strong relationship between increased BMI and lymphedema incidences (Soran et al., 2011). Increased body weight is a modifiable risk factor according to Cheville et al, primary prevention of lymphedema remains underemphasized. Identifying modifiable risk factors e.g. obesity will improve primary prevention care (Goffman, Laronga, Wilson, \& Elkins, 2004).

We found that $44.1 \%$ of patients $(n=15)$ have no report regarding their nodal status at diagnosis; this displays how deficient are our records. $32.4 \%$ of patients $(\mathrm{n}=11)$ had $\mathrm{N} 1$ status at diagnosis $8.8 \%$ had N2 or N3 at diagnosis. However this result is not conclusive due to the deficiency of the data. The recording of the stage at diagnosis was much more deficient was very difficult to analyze. $70.6 \%$ of patients $(n=24)$ underwent axillary clearance, this is in accordance with the literature (van der Veen et al., 2004).

Axillary lymph node dissection is a known major risk factor for lymphedema (Campisi, 2002). However, according to the reports, no patient underwent axillary sampling, but the histopathology for some patients proved that they did not undergo proper axillary clearance as we will discuss later. $23.5 \%$ of patients $(n=8)$ did not undergo axillary operation at all for these it might be the tumor infiltration or the RT are the main risk factor for lymphedema.

Upon studying the type of breast surgery, $58.8 \%$ of patients $(n=20)$ underwent modified radical mastectomy while the others either did not undergo surgery $(n=4)$ or underwent simple mastectomy $(n=4)$ or underwent wide local excision $(n=5)$; one patient underwent breast surgery but lost her report. This may give us some idea about the higher incidence of lymphedema if the patient undergo modified radical mastectomy, although in one studythe type of breast operation was not found to be a significant risk factor for lymphedema.

No breast reconstruction was done for all the thirty four patients. This is in accordance with the reported studies wherein breast reconstruction was found to be a protective factor against lymphedema (Lopez Penha et al., 2014). Four patients did not undergo any operation the lymphedema was caused most probably by the tumor infiltration in this situation. 
Upon analysis of the number of extracted affected lymph nodes, we excluded the patients who didn't undergo axillary operation $(n=8)$ those who lost their histopathology report $(n=5) .52 .4 \%$ of the remaining patients $(n=11)$ had $<10$ lymph nodes removed during axillary operation. Only one patient had $>30$ lymph nodes removed from her axilla while $26.4 \%(\mathrm{n}=9)$ had 10-29 lymph nodes removed from their axillae (Han et al., 2012). It was found in the previous studies that lymphedema incidence increases with the increased number of removed lymph nodes, but this does not seem to be the case in our study.

The number of affected lymph nodes in our study was $>10$ only in $23.8 \%$ of patients $(n=5)$, this reflects that increased no of affected lymph nodes does not seem to increase the LE risk. Upon reviewing the literature two studies were found, one Iranian study found that the increased number of affected lymph nodes is a significant lymphedema risk factor; another study found it not significant risk factor (Kosir et al., 2001; Vignes, Arrault, \& Ebelin, 2006). We found that $38.2 \%$ of patients $(\mathrm{no}=13)$ were offered only supraclavicular RT. The remainder have lost their records, received supraclavicular (SC) posterior axillary (PA) RT or received PA radiotherapy only. RT to the axilla is a well-known risk factor. In one study the SC radiotherapy specifically was a major risk factor. This seem to be the case in our study because $47 \%$ of patients (no=16) received SC radiotherapy, either alone or in addition to PA radiotherapy.

$44.1 \%$ of the patients $($ no $=15)$ did not undergo $\mathrm{RT}$ at all, in these patients it might be the ALND or tumor infiltration the major LE risk factor. All patients presented with arm swelling. Additionally, $70.6 \%$ of patients $(n=24)$ presented with arm heaviness whereas $26.5 \%(n=9)$ presented with arm pain. Patients were offered different treatment modalities like exercise, limb elevation bandaging, but some patients were not offered any treatment they do not know where to find help. Custom made compression garments are not available in Sudansome medical supplies centers are modulating lower limb garments to be used for the upper limb. These garments are expensive, and this is another problem which faces lymphedema patients. Compression using crepe bandage, with thin layer of cotton beneath it is another treatment option. Some poor patients could not even afford the crepe bandage although it is much cheaper than the compression garment. Most of these problems are found even in the developed countries; in Australia a study was conducted and concluded that the general practitioner has an important role in detecting lymphedema, advising where treatment can be accessed which schemes might assist financially (Marcos et al., 2012). Another new Canadian study pointed to the fact that although the number of individuals coping with chronic edema/ lymphedema continues to increase, the disparity between diagnosis, treatment funding across Canada endures ( $\mathrm{Lu}$, Hong, Chou, \& Hsiao, 2015). The reasons for this include a lack of public awareness of the condition insufficient education knowledge among health care providers regarding aetiology management limited financial coverage to support appropriate methods materials.

Only one patient was referred to a physiotherapistand this reflects that lymphedema is neglected by most doctors. The remainder patients were taught by the general nurses or the oncologists. We have no specialized lymphedema physiotherapists or nurses. In an Australian studythe authors concluded that oncology nurses are ideally positioned to monitor women for early signs of swelling to advice women on the range of treatments available (Marcos et al., 2012). This is in contrast to our general nurses working at the oncology clinics and even though, only a minority of them is giving advice to the LE patients. Upon analysis of the effect of elevationit was effective in $90.5 \%$ of the compliant patients, while exercise was effective in $84.2 \%$ of the compliant patients. All treatment options were offered to patients after development of the LE. Elevation and exercise education can be started after operation for the patients at high risk for LE development or even preoperatively. In a prospective study there was a lower incidence of arm morbidity, including reduction in arm circumference after preoperative education and early physiotherapy. It was found that $100 \%$ of the compliant patients for bandaging said that it is effective, whether they were instructed by the researcher or somebody else. This may reflect that bandaging is more effective than exercise and elevation, although in a previous studyit was stated that management of LE is extremely variable, currently there are no standard recommendations. This lack of information results in inadequate or delayed management. The reduction in the arm circumferences was really appreciable although only four patients were followed up; that seems to be a quite small number but we can understand more that it is effective when we know that most of patients did not come back for the follow up because they felt better and no need to come back. Most of our compliant patients said bandaging is more effective than elevation or exercise alone.

\section{Conclusions}

Lymphedema following breast cancer treatment continues to be a significant long-term morbidity in the current era. Postoperative radiotherapy to axilla, presence of comorbid conditions are significant risk factors for lymphedema development. Since there is no ideal treatment available for established lymphedema future efforts should be focused on optimizing treatment combinations, evolving minimally invasive methods like sentinel node biopsy for staging axilla. In most of our patients, bandaging is more effective than elevation or exercise alone.

\section{References}

[1] Ahmed, A. A. M. (2014). Clinicopathological profile of female Sudanese patients with locally advanced breast cancer. Breast disease, 34(3), 131134. http://dx.doi.org/10.3233/BD-140363.

[2] Alawad, A., Alshiekh, A., \& Alhaj, A. (2014). Demographic Characteristics and Review of Patients with Locally Advanced Breast Cancer in Sudan. Afrimedic Journal, 4(2), 5-8.

[3] Alawad, A. A. M. (2014). Evaluation of clinical and pathological response after two cycles of neoadjuvant chemotherapy on sudanese patients with locally advanced breast cancer. Ethiopian journal of health sciences, 24(1), 15-20. http://dx.doi.org/10.4314/ejhs.v24i1.2.

[4] Campisi, C. (2002). [Surgery of breast cancer, secondary lymphedema and quality of life: a challenge for the general surgeon]. Ann Ital Chir, 73(5), 471.

[5] Goffman, T. E., Laronga, C., Wilson, L., \& Elkins, D. (2004). Lymphedema of the arm and breast in irradiated breast cancer patients: risks in an era of dramatically changing axillary surgery. Breast $\mathrm{J}, 10(5)$, 405-411. http://dx.doi.org/10.1111/j.1075-122X.2004.21411.x

[6] Han, J. W., Seo, Y. J., Choi, J. E., Kang, S. H., Bae, Y. K., \& Lee, S. J. (2012). The efficacy of arm node preserving surgery using axillary reverse mapping for preventing lymphedema in patients with breast cancer. $J$ Breast Cancer, 15(1), 91-97. http://dx.doi.org/10.4048/jbc.2012.15.1.91.

[7] Kosir, M. A., Rymal, C., Koppolu, P., Hryniuk, L., Darga, L., Du, W., et al. (2001). Surgical outcomes after breast cancer surgery: measuring acute $\begin{array}{lllll}\text { lymphedema. } J & \text { Surg } \quad \text { Res, } & \text { 95(2), } & 147-151\end{array}$ http://dx.doi.org/10.1006/jsre.2000.6021.

[8] Lopez Penha, T. R., Voogd, A. C., Heuts, E. M., Ijsbrandy, C., Hendrix, N. A., von Meyenfeldt, M. F., et al. (2014). Reduced prevalence of lymphedema in patients with reconstructive breast surgery. Breast J, 20(6), 671-673. http://dx.doi.org/10.1111/tbj.12342.

[9] Lu, S. R., Hong, R. B., Chou, W., \& Hsiao, P. C. (2015). Role of physiotherapy and patient education in lymphedema control following breast cancer surgery. Ther Clin Risk Manag, 11, 319-327.

[10] Marcos, A. L., El Gaaied, A. B., Ayed, F. B., Hassen, S. B., Zervoudis, S., Navrozoglou, I., et al. (2012). Lymphedema of the arm after surgery for breast cancer: new physiotherapy. Clin Exp Obstet Gynecol, 39(4), 483488.

[11] Monleon, S., Murta-Nascimento, C., Bascuas, I., Macia, F., Duarte, E., \& Belmonte, R. (2014). Lymphedema Predictor Factors after Breast Cancer Surgery: A Survival Analysis. Lymphat Res Biol. http://dx.doi.org/10.1089/lrb.2013.0042.

[12] Soran, A., Wu, W. C., Dirican, A., Johnson, R., Andacoglu, O., \& Wilson, J. (2011). Estimating the probability of lymphedema after breast cancer surgery. Am J Clin Oncol, 34(5), 506-510.

[13] van der Veen, P., De Voogdt, N., Lievens, P., Duquet, W., Lamote, J., \& Sacre, R. (2004). Lymphedema development following breast cancer surgery with full axillary resection. Lymphology, 37(4), 206-208.

[14] Vignes, S., Arrault, M., \& Ebelin, M. (2006). [Poor influence of surgery on upper limb lymphedema volume in patients after breast cancer treatment] $J$ Mal Vasc, $31(4$ Pt 1), 202-205. http://dx.doi.org/10.1016/S0398 0499(06)76544-1. 